too great distension has taken place, or peritonitis has not supervened.

After the tenth day I usually give strychnin and continue to convalescence and during the same, then dilute hydrochloric acid in place of salicylate of ammonia in three or four days after fever disappears.

Administration of food begins after the sixth, eighth or tenth day; the first choice is always milk-sweet milk, or good fresh buttermilk; fruit juices from first to last. The patient will often relish some kind of fruit juice at the first. It is not a food in one sense, but avery one knows its beneficial influence in sickness or healin. It does not derange the stomach or bowels; it refreshes. makes the stomach better, and helps the patient in ways that we know nothing about, physiologically. It ought always to be given as a rule with but few exceptions. Do not use a bedpan. Let the patient get up. My patients get up themselves; they know what they are about; they are not too weak. Do not let that bugbear, perforation, scare you out of your wits. It does not often occur; I practiced thirty-two years with two cases only. It helps the patient to get up, gives him an airing, changes his position, and he feels better afterward. $\mathrm{I} \mathrm{am}$ satisfied that it arouses his vital energies, and is a factor in preventing his getting into that dormant state which I saw so frequently in my early practice.

Now for the results: The thirty-first case died from overeating and eating improper food on the fifth day of convalescence. The patient was a young woman with her first child, 10 months old, and had poor health from the time of its birth; was anemic. Overeating caused indigestion, sick stomach, vomiting, diarrhea, prostration and death, but no, return of fever.

The sixty-second case died on the twenty-second day, from hemorrhage. Pneumonia ensued on the fifteenth day. The patient was a young man, 22 years old.

The seventy-third case was a young man who was convalescent on the fifteenth day; got up, went to the cupboard, ate too much, and had a relapse. On the twentyfirst day, when he was better again, perforation took place on the evening of that day, which caused his death. In the ninetieth case, the patient had a relapse three wecks after the first attack, with perforation on seventh or eighth day of relapse. He had been treated by another

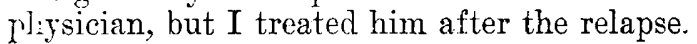

In the one hundred and tenth case I was not called until the eighth day. The patient was unconscious, and death took place three days afterward. I did not have a chance to even commence treatment. It is hardly fair to count this case, but I have done so.

The sixth and last case was a married lady, about 45 years of age. She was the tallest and most slender woman I ever saw. She seemed overcome by the disease from the start, and did not have any rise of temperature but one day. She was really the weakest mortal I ever treated, and the sequel showed she really had no vital resistance, as she died on the eighth or ninth day with typhoid fever; when she had no fever.

I seaving out the one hundred and tenth case, which it is surely not fair to count, so far as treatment is concerned, this would show 3.5 per cent. of deaths.

Now, while I might have occasion to revise my statistics in the future in a way which would not make so favorable a showing, my opinion is that I could make a more favorable percentage even than I have made.

Of three things I am certain: that the treatment will greatly mitigate the symptoms, will shorten its duration, and greatly lessen the danger of death.
Whoever will may decry the medical treatment of typhoid fever as much as he pleases. I am sure of its influence, and look upon it as an established fact in medical science, and when called to a case, undertake the treatment without any apprehension as to the result. Whenever we can arrive at this state of mind in a dis. ease like typhoid, which carries off its thousands of the youngest and fairest, with the highest hopes and the brightest prospects, then we have accomplished something for ourselves and for humanity of which we may well be proud.

\section{ACETANILID IN TYPHOID FEVER.}

\author{
WITH A REPORT OF SIX CASES.* \\ BY EDMUND C. BRUSH, A.M., M.D. \\ ZANESVILLE, OHIO.
}

The physician who has been in active practice for twenty-five years and followed the current medical literature has read of many cures for consumption and of methods of aborting typhoid fever. He has also read of many methods of treating typhoid fever and perhaps has tried some of them. I have never believed that typhoid fever could be aborted; I have never seen it aborted. Long ago I seitled down to the idea that good nursing, judicious feeding and medicines to meet symptoms, as they arise, is the best plan of treatment. As you all know, same cases defy any and all methods of management and run a vicious and seemingly unmanageable course.

My home is in Zanesville, Ohio, situated in the Muskingum River ralley, where typhoid fever is with us always; varying in frequency in different seasons of the year and also in different years. The severity of the type also varies in the same way.

The prime object in any plan of treatment is to control the fever; the severity of a given case and its outcome depending largely on the height of the temperature. It is not proposed to mention even the many plans, past and present for accomplishing this end.

The purpose of this paper is to report six cases in which acetanilid was used to control the fever. These are not the only cases in which I have used that drug, but the six in question were treated in a hospital, where records could be kept. They.occurred at the same time, and all originated at Camp Thomas, Chickamauga, Ga., during the latter part of August and early in September, 1898. The patients were soldiers belonging to one erganization and of an average age of 22 years. They were sent home from camp with the disease in various stages. Two had rose-colored spots, and all had either diarrhea or a tendency to it, when admitted to the Zanesville Hospital. One had a persistent cough with sore throat. Three suffered at times with epistaxis. All had distended abdomens, but in only two cases was it pronounced. Not one had any hemorrhage from the bowels. The shortest run of the fever was twentyfour, the longest thirty-five, and the average thirty days.

In addition to the six cases reported, I saw ten others among the same body of men. These were taken care of in their respective homes by home people. I think it is generally known that the typhoid fever in the camp mentioned was not of a mild type. One of the ten cases, seen by me outside of the hospital, died in the fourth week of the disease, from persistent and violent hemorrhage from the bowels. All the others recovered.

(ASE 1.-Tongly, aged $1 \%$ years, was probably in the

* Presented to the Section on Practice of Medicine, at the Fiftieth Annual Meeting of the Ameriean Medical Association, held at Columbus, Ohio, June 6-9, 1899. 
tenth day of disease when received. He had rose-colored spots on the abdomen, and diarrhea when admitted. There was no delirium and but slight distension of the abdomen. He had fever for fourteen days after admission, the average morning temperature being 99.6 ,; average evening temperature, 101. At noon on the day after admission his temperature was 104.

CASE 2.-Tucker, aged 25 years, was probably in the eighteenth day of disease when received. The rose-colored spots disappeared soon after admission. $\mathrm{He}$ had epistaxis on the twenty-second and twenty-eighth days of disease and a troublesome cough throughout, with mild diarrhea and slight distension of the abdomen. He had fever for twelve days after admission, average morning temperature being 99.2 ; average evening temperature, 100.4. The highest temperature was 103 , on evening of the twenty-third day. 'This patient had a decided yellow tinge to his skin and conjunctiva during the whole course of the fever.

CASE 3.-Trump, aged 21 years, was probably in the seventh day of disease when received. The rose-colored spots were well marked, and there was considerable delirium with subsultus tendinum when admitted. $\mathrm{He}$ had some diarrhea, and abdomen was distended. On the sixteenth day, after using acetanilid, his temperature at $6 \mathrm{p}$. $\mathrm{m}$. was 98 , and he complained of feelin chilly, He had fever for twenty-four days, average morning temperature being 100 ; average evening temperature, 101. The highest temperature was 103.2 , on the evening of the eighteenth day. This patient had a pronounced yellow tinge to his skin and conjunctiva throughout his sickness.

CASE 4.-Rambo, aged 22 years, received about the ninth day of the disease. He had a troublesome cough and a persistent sore throat; was nervous, delirious and could not sleep; had subsultus tendinum. Some diarrhea and vomiting occurred at times, with well-marked distension of the abdomen; severe epistaxis on the thirteenth, eighteenth, twentieth and twenty-sixth days of disease. In the fourth week he had severe conjunctivitis, and had fever for twenty days after admission, the average morning temperature being 100.8; average cvening temperature was 101.8. The highest evening temperature was 103.4, on eleventh day.

This was the only case in which acetanilid was used in loses larger than three grains. For the first three days after his admission this patient's temperature was hard to control, so that the dose was doubled and six grains was given, and on several occasions during those three days, this dose was repeated in one hour because the first dose did not produce any perspiration or reduction of temperature. After the third day of his admission and the twelfth day of the disease, the temperature was controlled with three-grain doses.

CASE 5.-Wortham, aged 25 years, was received near the beginning of attack. He had diarrhea all through sickness, but easily contruled, and had some cough and nausea at times. Late in the disease he suffered from insomnia; this was relieved with sulphonal. This man had a relapse due to tobacco brought in surreptitiously. He had fever thirty-five days, including relapse, the average morning temperature being 99.4, average evening temperature 101. The highest evening temperature was 103.4 , on the eighteenth day.

CASE 6.-Hahn, aged 21 years, was received on the third dav of the disease. He had well-marked abdominal distension, with diarrhea, followed in the second week with constipatiol: : had epistaxis on twelfth, thirteenth and fifteenth days. He had no delirium when awake, but talked a grod deal when slceping; had fever for twenty-eight days, the avarage morning temperature being 100.8, average evening temperature 101.8. The highest evening temperature was 103.4 on the twelfth dity. 'This man was raised in our Children's Home, and during his sickness was home-sick. Frequent visits from the matron of the Home helped the nostalgia.

On several occasions he complained of feeling chiliy after taking acetanilid. Calomel followed by castoroil was given for the constipation and had to be used a number of times. Whenever this patient's bowels did not move almost daily ho complained of distress. This distress was always relieved by a laxative, and the patient said he felt better after it. As the pulse and respiration in all the cases were in ratio with the temperature, ther are omitted.

The foregoing report shows that the cases were typically typhoid and not of a mild character. Three of them were in the first week, two were in the second week and one in the third week of the disease when they came under my charge. All had diarrhea at times, of more or less severity. Milk with lime water formed the chief article of diet, varied with malted milk, beef broin, whites of eggs, ice cream and corn-meal gruel. Ice and water were not restricted. The distended abdoriens were freely anointed with turpentin. Antiseptic mouth washes were used. For the diarrhea, a tablet consisting of opium and camphor each $1 / 4$-grain, ipecac $1 / 8$ grain and acetate of lead $1 / 6$ grain was given. Bismuth or essence of pepsin generally relieved the nausea or vomiting. When a laxative was needed, calomel was administered, followed by castor-oil with a few drops of laudanum.

As many of the comrades of these men suffered from malarial troubles, it was deemed best to use some quinin in these typhoid cases. This was given in two-grain doses three times a day, or with the acetanilid. No whiskey was used. All were given a cold sponge-bath every morning.

Acetanilid was the antipyretic used, and it was efficient and effective. The directions given the nurses were to give three grains of acetanilid every three hours whenever the temperature was over 101, until it fell to that point or below. If, after giving a dose, the temperature showed a decided tendency not to fall, a second dose was given in two hours.

As an illustration, the following is taken from the record of one of the cases reported. At 5 a. $\mathrm{m}$. his temperature was 102.6. Three grains of acetanilid was given at once and this was followed by free perspiration. In two hours the temperature was 100.6 and only one dose had been given. At 2 p. m. the temperature was up to 103; acetanilid was again given and in two hours the temperature vas 101 , and did not go above that point that evening.

Nearly all patients broke out into a good perspiration after the first dose, and the temperature began to decline; the second dose made the perspiration more free and the decline in the temperature more decided. In every case there was comparative ease in controlling the fever by the method given. There was no effort to reduce the evening temperature below 101. It often went below that point, and the patients would only complain of being chilly. There were no bad effects noted from the use of the drug. It was not given in large doses, it was not given often. Frequently two doses in one day were all that a patient required. When, however, a high temperature persisted, the drug was used until the fever gave way. The nurses showed apti- 
tude in administering the acetanilid as soon as the idea of its use was explained; instructions were carried out carefully.

$I_{\iota}$ is to be remembered that these patients were robust young men, that the feeding and care given them was systematic, and they never questioned what was to be done.

I do not present this paper because I think that I have found the treatment for typhoid fever, but because in using acetanilid, I had success, and I believe that, used with judgment, it is a valuable remedy in treating typhoid fever. Please observe that no attempt was made to produce a sudden fall in the temperature by using large coses. I preferred to give small doses and by repeating them produce a gradual decline in the fever. Excepting one case, three grains was the largest dose given.

Furthermore none of the remedies used were given unless their need was clearly indicated. The whole idea was not to medicate, but to pilot the patients through the rapids of the disease and to land them on the shore of recovery.

DISCUSSION ON PAPERS OF DRS. BOONY. TAYLOR, ROUSCH AND ERUSH.

Dr. J. A. Witherspoon, Nashville, Tenn.--This subject is one of the most important that we have to deal with. If I understood the gentleman correctly, he stated that malarial fever had about died away, and that most fevers today were of typhoid origin. Now, gentlemen, the differences in the treatment of typhoid fever in recent years has made it evident, in my humble judgment, that the fever mentioned is a different type of fever altogether. I do not know what you have in this part of the country, but I do know that we have in Tennessee, and in that section of the country, a fever that has none of the etiologic or elinical factors of typhoid fever or malaria. We never find, after the tenth day, the typhoid bacillus in the stools, or the malarial plasmodium in blood. We never have the nervous symptoms of typhoid fever. The temperature goes up suddenly without any incubation period. With the fever there is at once great prostration. It is a fever with leaky skin, and not the dry skin of typhoid; with general abdominal tenderness and the scaphoid belly; with rather constipated bowels, with small scybala actions at first, and not the mushy stools of typhoid fever; a fever that runs a rather low and favorable course if not overtreated; a fever without enlargement of the spleen and liver; one where the effect on the nervous centers is very slight; one with markedly fluctuating temperature. It takes one general purge at first, in these cases. I can not imagine any worse treatment than giving an active purgation of 3 to 6 grains of calomel every day for six days to get up a stool as frequently as one wants to, as mentioned. In typhoid fever we have an irritation in the intestinal canal, of the solitary follicles, an inflammation of Peyer's patches with ulceration, and by this active purgation you absolutely add fuel to the fire, running the risk of the formation of a quick slough. In my limited experience with eases of typhoid fever, 1 believe they do better when the bowels are moved with a gentle enema, ratner than by a purge. I do not believe in continuous constipation, but I do believe in enemas, and not in the use of purgatives at all. Another point: there is no treatment of typhoid fever to be applied to every case. We should meet the conditions as they arise. If I should select any one treatment, it would be cold water inside and outside. It is by far the safest and best treatment ever introduced. It is not the treatment of the aisease, but the treatment of the patient. One should earefully consider the vitality of the patient and treat according to the symptomatology.

The gentleman who read the last paper stated that the essential element in the treatment was to control the fever. Why, the toxemia is the dangerous thing we have to deal with. The toxins are sent out into the system and overwhelm the nervous centers. The fever plays but a little part. If any man thinks that the Brand method of treatment is for the purpose of lowering the temperature only, he has a wrong conception of it. The temperature plays but a little part. As to acetanilid, I want to place myself on record as saying that I do not think that any of the coal-tar derivatives have a place in the treatment of typhoid fever. Any drug that lowers the resisting powers of the patient and acts on the heart muscle itself, should have no place in the treatment of typhoid fever. There- fore, I oppose any coal-tar derivatives. The best treatment is rest, water, regulation of the diet, careful nursing, regulation of the bowels, and, if you do not treat too much, the vast majority of the cases will get well.

DR. C. H. Mills of Illinois-I have been in the habit of treating typhoid fever since 1856 with rest, feeding with toast. water, some milk and six drops of turpentin made into an emulsion and given every three or four hours; this is the treatment of Dr. G. B. Wood of Philadelphia. I advise occasional enemas of either water, or water and turpentin. Occasionally typhoid fever is modified by the presence of malarial fever, and this is usually spoken of as typhomalarial fever. It is a combination of the two diseases. Where this occurs I give quinin, 60 grains in twenty-four hours, which eliminates the malarial part. In ordinary practice I can not use the cold bath. This use of cold water was common when I commenced practice and is common to-day. Turn the water on the wrist and on the head and do not disturb your patient. The great trouble is that we interfere too much with the patient. Give rest in a well-ventilated room.

Dr. Crook, of Tennessee-I only wish to add to Dr. Witherspoon's statement. I find that he gives it very nearly right. If 1 am called to treat a suppurating wound, I use drainage and antisepsis. If I am called to see a case of typhoid fever, which means that the alimentary canal swarms with bacilli, I use drainage and antisepsis.

DR. L. I. MatTHEWS, Joplin, Mo.-I thoroughly agree with the remarks made by Dr. Witherspoon. I have had the same serious doubt, in reference to the type of fever we have in the West that Dr. Witherspoon seems to have. He did not say that we have a special type of fever, which differs from malarial fever or typhoid fever in the West; he did not say that, but I think he believes it. I know that I believed it for many years. Many years ago $I$ read a paper that demonstrated conclusively, to my mind at least, that what most of the physicians were calling typhoid fever was some peculiar, distinct disease different from typhoid fever, it being neither typhoid nor malarial. Soon after I had a number of these cases, in which the last four running much the same course, developed fatal intestinal hemorrhage. While I believe, in the West, we do not have the typical cases of typhoid, as described in text-books, I now believe that all of them are due to the bacillus typhosus. My experience has taught me to pay but little attention to the fever. If I am called to sec a case with remarkably high temperature, and yet with a good pulse, I feel secure; but if I am called to see a case with low temperature, with quick, rapid pulse and with indications that the disease is going to be one of prostration, with probably heart failure, I then am apprehensive. Pay attention to the heart, rather than the temperature.

Dr. Scotr of Iowa-It has been my opinion that the origin of the bacteria in infectious diseases in general is from outside the human body. The illustration of the endemic of typhoid fever, as given by the author of the first paper, is evidence of this fact. It is developed from some ontside source, as decomposing vegetable or animal matter. The symptoms of typhoid fever are due to toxins which are developed within the cells, which, being absorbed by the body, produce effects on that body, especially the nervous system. The treatment of infectious diseases in general, and typhoid fever in particular, must necessarily be the removal of the toxins from the system. The fever should be treated by removing its causes, i. e., its toxins. This may be eliminated: 1. By the use of water internally, so eliminating it through the kidneys. 2. By the external use of water. 3. By action on the liver, which is the organ, when properly stimulated, that destroys or reduces the amount of toxins and so gets rid of them. 4. By the use of intestinal antiseptics. 5. By nourishment. The administration of nourishment from the beginning of the disease is necessary. In regard to getting up for stool, there should be no hard and fixed rule in this matter. During the first week the patient should be allowed to get up, but not during the last stages, when there is danger of perforation of the bowels. I do not believe that acetanilid has any effect in this fever. It seems to me that the giving of any arug should depend on what other agent is given with it; acetanilid, in small doses, given with caffein or whisky, may be valuable.

Dr. Herrington of Ohio-It will be a long time before we settle how to treat typhoid tever. The fact that we treat typhoid by different means shows that there are a lot of things we do not know. But $I$ hope and trust that every physician will aspire in the treatment of typhoid fever to the extent of trying to abort the fever and not to that of curing it. The question to-day is, can typhoid fever be aborted? I am among those who claim that it can be aborted. Most of you expect to have a low muttering delirium, tympanites, ete. To prevent 
this, commence early in the disease, with mild catharsis, using small doses of $1 / 60,1 / 80$, or even $1 / 960$ of a grain of ealomel and give it every few ninutes, and so keep up a light-colored abnormal discharge from the bowels and try to bring the discharge to the normal. The liver is not acting properly and the discharge comes at first from the lower part of the bowel. This prevents the tympanites and aborts the disease in quite a large majority of the cases in not over fifteen days.

Dr. LouIs BISHOP, New York City-This discussion covers the treatment of typhoid fever thoroughly. I would like to make a phrophecy that the time is not many years distant when we will have an efficient antitoxin treatment. It has been shown by experimental means that typhoid fever can be prevented by the use of the antitoxin made from the typhoid bacillus. Dr. Welch of Johns Hopkins Hospital, Baltimore, in an address before the New York Academy of Medicine, gave the details of the work of bacteriologists, showing that the time is not more than a few years distant when we shall have an antitoxin treatment for typhoid fever. At present I believe that the best treatment for this disease is the cold water treatment, externally and internally. I have measured the urine in patients who have been subjected to the cold bath, and I have seen it about double itself as soon as the treaiment was placed in operation. I believe that ihe success in the cold bath is largely due to its elimination of the toxins.

DR. J. C. WILson, Philadelphia-It is clear to me that such an interchange of opinion as this must be of more value than we at the moment realize. The interchange of opinion and experience of practitioners living at distant points and in different parts of the country is most useful.

I may be permitted to speals of our experience in the German Hospital in Philadelphia, where we have always a great many cases of enteric fever, and during the recent outbreak had as many as sixty-eight eases in the wards at one time In that institution the cases are treated systematically by cold bath ing, according to the method of Brard, but under no circumstances do we use the internal antipyretics. In theory our object is to elininate the toxins of the disease as rapidly as possible, and we avoid introlucing into the blood-current toxic drugs, such as acetanilid, phenacetin, etc., the perturbating effects of which are so marked.

Brand and all those who have carefully carried out his treatment on an extended seale concur in the view that the cold bath treatment is not of itself an antipyretic method. The reduction of teinperature is one of a group of effects produced by systematic cold bathing. Another effect to which too little attention is paid is the action of the cold bath on the kidneys as organs of elimination-the toxic coefficient of the urine after the bath having been demicnstrated to be inereased from five to six times.

Albuminuria is inuch more common and more marked in cases treated by systematic cold bathing, than by the expectant method, but it is a transient condition, disappearing with the recovery, and in this respect resembles the albuminuria of jellow fever.

I desire to speak of our experience with reference to the posture of the patient in bed during the attack. We have alopted the plan of having the bath-tub stationary in our small fever wards and, due consideration being had to the condition of individual patients, allowing them to walk to the tub with the assistance of the attendants. In this way the patient is aroused from his recumbent posture regularly every third hour, walks to the bath or is carried there by the attendants, and returned to his bed afterward. We have practiced this plan since Jan. 11, 1897, and the results have been entirely satisfactory. In 165 eases of enterie fever treated during the year 1897 the mortality was 6.5 per cent.

Dr. Jenkins of Iowa-More than fifteen years ago I was satisfied that house flies were the greatest carriers of the contagion, and during the late war we had much typhoid fever in the camps, brought on by house flies. The excreta should be destroyed. We could not put soldiers in a more perfect hotbed for the development of the disease than we did in the late war. They were placed in the camps, and when they were taken with typhoid fever they were removed to the hospital; from the hospital they went to the camp, and so carried infection. Good water was to be had, but nearly everybody who was susceptible to typhoid fever was infected in these camps. This is a very important thing, and the medical profession should speak out and see that no such thing should again happen.

Dr. George Boodr, Independence, Iowa-In connection with the external use of water, it should be given in regularly prescribed quantities internally. During the last epidemic I carefully watehed Widal's reaction day after day. in all my cases, in a number of cases treated by neighboring physicians who wished their diagnoses confirmed, and in one case from Jack- sonville, Fla., a specimen of blood having been sent to me by a nurse in my service who went to the camp there; and as I saw in each case the same reaction, the bacilli clumping and perishing in a short time before my eyes, I could not help thinking that in the not distant future there would be a typhoid serum or antitoxin which would give the same results in the prevention of typhoid fever as we now obtain by the use of antitoxin in diphtheria.

Dr. L. F. Rousch, New Haven, W. Va.-I wish to speak in reference to what the gentleman from Tennessee said about the cathartic action of medicine. He made it appear that $I$ used calomel in doses Iarge enough to produce an irritative' effect. While it is well enough to use large doses, as advised, it is not continued.

\section{PERIOSTEAL CARIES FROM BACTERIAL ORIGIN.* \\ M. H. FLETCHER, D.D.S., M.D., M.S. CINCINNAT, OHIO.}

Of recent years my attention has been called to a number of cases of neuralgia, the exciting causes of which were obscure, as is frequently the case. Among others was a case of supposed tic douloureaux accompanied by the apparent death of the periosteum over a larger portion of the lingual surface of the inferior maxilla of the left side. While the exciting cause in this case may have been central, it apparently was peripheral; it yielded to local treatment, which fact strengthened belief in the peripheral origin. The finding of this lesion was the result of desperate effort, every logical treatment, both systemic and local, having been resorted to, aside from nerve section, and all without relief.

Case 1.-Mrs. X., aged 55 years, suffered with paroxysmal pains resembling tic douloureaux; these continued with increasing tendency for three or four years, always including the first molar, which was the only tooth remaining back of the first bicuspid, and it was finally extracted, after having been devitalized and properly treated. For a short period this gave some relief, but the paroxysms returned with increased severity.

There was nothing abnormal in the macroscopic appearance of the bone, flesh, or mucous membrane, and no tenderness nor swelling; no pus could be detected. Thinking that something abnormal might possibly be discovered with a magnifying-glass, one was used, and a tiny slit was found, not to exceed one-sixteenth of an inch in length, and situated on the summit of the alveolar ridge, at the former seat of the offending first molar. My first impression was that I should find here a portion of the root of a tooth, but none could be discovered; the probe, however, after being forced between the lips of the opening, went into a large pocket on the lingual surface of the bone without further resistance; careful probing showed this pocket to reach from the summit of the alveolar ridge to the inferior border of the jaw, and from the first bicuspid back to the angle, including the inferior dental foramen. The surface of the bone, instead of being rough and necrotic, had the feel of being emburnated and without periosteum; of course, the exact conditions of such cases can only be verified by post-mortem examination, and as yet no opportunity has offered for such investigation.

It is held by others that the periosteum in this case is probably still present, and either divided, or entirely adherent to the supervening soft tissues, and I believe that all known laws of pathology would support this theory. There are some facts, however, which go to support the hypothesis that the periosteum is dead, in this and all similar cases.

*Presented to the Section on Stomatology, at the Fiftieth Annual Meeting of the American Medical Association, held at Columbus, Ohio, June 6-9, 1899 . 